\title{
Hilbert Bases, Unimodular Triangulations, and Binary Covers of Rational Polyhedral Cones*
}

\author{
R. T. Firla ${ }^{\dagger}$ and G. M. Ziegler \\ Department of Mathematics, MA 7-1, Technische Universität Berlin, \\ Strasse des 17. Juni 136, 10623 Berlin, Germany \\ \{firla,ziegler\}@math.tu-berlin.de
}

\begin{abstract}
We present a hierarchy of covering properties of rational convex cones with respect to the unimodular subcones spanned by the Hilbert basis.

For two of the concepts from the hierarchy we derive characterizations: a description of partitions that leads to a natural integer programming formulation for the HILBERT PARTITION problem, and a characterization of "binary covers" that admits a linear algebra test over GF(2) for the existence of BINARY HILBERT COVERS.

Implementation of our test leads to interesting new examples, among them: cones that have a HILBERT PARTITION but no REGULAR one; a four-dimensional cone with unimodular facets that has no HILBERT PARTITION; and two five-dimensional cones that do not have any BINARY HILBERT COVER.
\end{abstract}

\section{Introduction}

The geometry of rational polyhedral cones and their Hilbert bases is of basic importance for subject areas that range from Integer Programming to Algebraic Geometry. So, Sebő's [13] HILBERT COVER conjecture posed in 1990 was motivated by considerations from Combinatorial Optimization; the first counterexample to the slightly stronger HILBERT PARTITION conjecture was given in the setting of Toric Varieties by Bouvier and GonzalezSprinberg [2].

Similarly, Sebő's main result, asserting that in dimension 3 REGULAR HILBERT PARTITIONS exist, was rediscovered and reproved by Aguzzoli and Mundici [1] as well as by Bouvier and Gonzalez-Sprinberg [3], who added an important uniqueness result.

\footnotetext{
* The second author was supported by a DFG Gerhard-Hess-Forschungsförderungspreis (Zi 475/1-2) and by the German Israeli Foundation (G.I.F.) Grant I-0309-146.06/93.

$\dagger$ Current address: Institut für Math. Optimierung, Otto-von-Guericke-Universität Magdeburg, Postfach 4120, D-39016 Magdeburg, Germany. robert.firla@mathematik.uni-magdeburg.de.
} 
Despite these investigations from different directions and with different motivations, a number of central questions remains open, among them, in particular, Sebő's HILBERT COVER and INTEGRAL CARATHÉODORY conjectures.

This note has three parts:

1. We present a refinement of Sebő's hierarchy that characterizes the covering properties of a rational cone with respect to the unimodular subcones spanned by Hilbert basis elements.

2. For two of the entries in this hierarchy we present characterizations that lead to computational procedures. Namely, the existence of a HILBERT PARTITION (as considered by Sebó) can be checked by solving an integer programming problem (see also [6]), while the existence of a BINARY HILBERT COVER (a new concept) is reduced to the solution of a linear system of equations over $\operatorname{GF}(2)$.

3. Implementations of these computational tests yield three new types of examples. So, we show that the Ohsugi-Hibi examples without a REGULAR HILBERT PARTITION do in fact have HILBERT PARTITIONS. We provide examples of simplicial cones in dimension 5 that do not have a BINARY HILBERT COVER, thus getting further than ever on the search for counterexamples in Sebó's hierarchy. We have an especially nice example of a cone in dimension 4, which has the hyperplane property (the Hilbert basis is "at height 1") and unimodular facets, and which has a BINARY Hilbert COVER, but no Hilbert PARTITION.

\section{Definitions and the Hierarchy}

All the cones $C \subseteq \mathbb{R}^{n}$ that we consider in the following are pointed, rational, and polyhedral, that is, they are sets of the form

$$
\begin{aligned}
C & =\operatorname{cone}\left\{a^{1}, \ldots, a^{m}\right\} \\
& :=\left\{\lambda_{1} a^{1}+\cdots+\lambda_{m} a^{m} \in \mathbb{R}^{n}: \lambda_{i} \in \mathbb{R}, \lambda_{i} \geq 0 \text { for } i=1, \ldots, m\right\}
\end{aligned}
$$

for rational vectors $a^{1}, \ldots, a^{m} \in \mathbb{R}^{n}$ such that $\{0\}$ is the largest linear subspace of $C$. A finite set of integer vectors $h^{1}, \ldots, h^{k}$ is a Hilbert basis of $C$ if each integral vector in $C$ is a nonnegative integral combination of $\left\{h^{1}, \ldots, h^{k}\right\}$, that is, if

$$
C \cap \mathbb{Z}^{n}=\left\{\lambda_{1} h^{1}+\cdots+\lambda_{k} h^{k} \in \mathbb{R}^{n}: \lambda_{i} \in \mathbb{Z}, \lambda_{i} \geq 0 \text { for } i=1, \ldots, k\right\} .
$$

The existence of a Hilbert basis for every cone was first established by Gordan (1873); van der Corput (1931) showed that the minimal Hilbert basis for pointed cones is unique: it is the finite set of nonzero integral vectors in $C$ that cannot be represented as a sum of two vectors of the same kind-see [12]. In the following, we exclusively refer to minimal Hilbert bases. We denote the minimal Hilbert basis of $C$ by $\mathcal{H}=\mathcal{H}(C)$.

The focus of this paper is to study the coverings of $C$ by certain families of simplicial subcones, that is, by cones $C^{1}, \ldots, C^{r}$ that are subsets of $C$.

Definition 1 (Interior Facets; Generic Points). Let $\mathcal{C}=\left\{C^{1}, \ldots, C^{r}\right\}$ be a set of subcones of a cone $C$. We call a facet $F$ of a subcone $C^{i}$ an interior facet if $F \nsubseteq \partial C$, where $\partial C$ denotes the boundary of $C$. We enumerate as $\mathcal{F}:=\left\{F^{1}, \ldots, F^{s}\right\}$ the set of all interior facets of the cones in $\mathcal{C}$. 
A point $g_{0} \in \operatorname{int}(C)$ is called a generic point (with respect to $\mathcal{C}$ ) if it is not contained in the boundary of any of the subcones $C^{i}$, that is, if $g_{0} \in \operatorname{int}(C) \backslash \bigcup_{j=1}^{s} F^{j}$.

Definition 2 (The Cover Hierarchy). Given any rational polyhedral pointed cone $C$ and a finite family of subcones $\mathcal{C}=\left\{C^{1}, \ldots, C^{r}\right\}$ each of the following properties implies the previous one.

COVER: $\mathcal{C}$ is a cover of $C$ if every point of $C$ is contained in one of the subcones $C^{i}$, that is, if $C=\bigcup_{i=1}^{r} C^{i}$.

BINARY COVER: $\mathcal{C}$ is a binary cover of $C$ if

(i) every generic point $g_{0} \in C$ is contained in an odd number of subcones $C^{i}$, and

(ii) every interior facet $F^{j}$ is a facet of an even number of subcones $C^{i}$.

PARTITION: A cover $\mathcal{C}$ is a partition if the intersection of any two subcones $C^{i} \cap C^{k}$ is a face of both cones, that is, if $\mathcal{C}$ forms a polyhedral complex.

REGULAR PARTITION: $\mathcal{C}$ is a regular partition if additionally the complex is given by the domains of linearity of a piecewise linear convex function on $C$. (For details on this concept see Chapter 8 of [14] and Chapter 5 of [15].)

BINARY COVERS have a homological interpretation: they correspond to generators for simplicial homology with $\mathrm{GF}(2)$ coefficients, that is, to generators of the relative singular homology group $\mathrm{H}^{n-1}(C \cap H, \partial C \cap H ; \mathrm{GF}(2))$, where $H$ is an affine hyperplane such that $C \cap H$ is a bounded, $(n-1)$-dimensional polytope.

A cone $C \subseteq \mathbb{R}^{n}$ is simplicial if it is generated by a linearly independent set of vectors. A simplicial cone $C$ is unimodular if it is generated by a subset of a basis of the lattice $\mathbb{Z}^{n}$, that is, if $C=$ cone $\left\{a^{1}, \ldots, a^{k}\right\} \subseteq \mathbb{R}^{n}$ for some set $\left\{a^{1}, \ldots, a^{n}\right\}$ of integral vectors with $\left|\operatorname{det}\left\{a^{1}, \ldots, a^{n}\right\}\right|=1$.

Proposition 3 (The Hilbert Cover Hierarchy). Let $C \subseteq \mathbb{R}^{n}$ be an n-dimensional pointed rational polyhedral cone, and let $\mathcal{U}=\left\{C^{1}, \ldots, C^{s}\right\}$ be the (finite) set of all $n$-dimensional unimodular subcones of $C$ that are generated by a subset of the Hilbert basis $\mathcal{H}=\mathcal{H}(C)$.

Each of the following properties of $C$ implies the following ones:

REgular Hilbert PARTITION: Some subset $\mathcal{C} \subseteq \mathcal{U}$ is a regular partition of $C$.

Hilbert PARTItion: Some subset $\mathcal{C} \subseteq \mathcal{U}$ is a partition of $C$.

BINARY HiLbert COVER: Some subset $\mathcal{C} \subseteq \mathcal{U}$ is a binary cover of $C$.

HILBERT COVER: $\mathcal{U}$ is a cover of $C$.

INTEGRAL CARATHÉODORY PROPERTY: Every integral vector $x \in C \cap \mathbb{Z}^{n}$ can be written as a nonnegative integral combination of at most $n$ elements of the minimal Hilbert basis $\mathcal{H}(C)$.

Incidentally, it seems that the Algebraic Geometry discussion started at the "high end" of this hierarchy, where the existence of a REGULAR HILBERT PARTITION is equivalent to the existence of a smooth projective torus-invariant resolution of the associated affine toric variety, see [2], [3].

In contrast, the Combinatorial Optimization motivation starts with the INTEGRAL CARATHÉOdory Property at the "low end" of the hierarchy. Thus, Sebő's [13] "Conjecture A" states that every cone $C$ has the INTEGRAL CARATHÉODORY PROPERTY: we call 
this the INTEGRAL CARATHÉODORY CONJECTURE. The stronger "Conjecture B" states that every cone $C$ has a HILBERT COVER: we call it the HiLBERT COVER CONJECTURE. Both conjectures are still open, but we contribute here as main (negative) results that

- In dimension $n=4$, there are cones that have a BinARY Hilbert CovER, but no Hilbert PARTITION: see Example 8.

- In dimension $n=5$, there are cones that have no BINARY HILBERT COVER, but which seem to have HILBERT COVERS: see Example 11.

- In dimension $n=10$, there is a cone that has a HILBERT PARTITION, but no REGULAR HiLBERT PARTITION: see Example 12.

(From any of these examples, it is easy to derive similar ones in any higher dimension.)

On the positive side, Sebó [13, p. 447] observed that for $n \leq 3$ one always has a HILBERT PARTITION. In fact, we know that there is even a REGULAR HILBERT PARTITION for $n \leq 3$, which is essentially unique.

Our goal was to test the properties of the Hilbert hierarchy for concrete examples. For this, the following section develops an Integer Programming model that in practice is quite effective for testing the HILBERT PARTITION property. While the HILBERT COVER property seems quite difficult to test algorithmically, we find that the BINARY HILBERT COVER property, which interpolates between HILBERT PARTITION and HILBERT COVER, is easy to test: in the following we reduce it to the solution of a linear system of equations over GF(2).

\section{Characterization Theorems}

In this section we present characterizations of BINARY COVERS and PARTITIONS that yield algorithmic approaches for computing such covers.

The following characterization of partitions and the integer programming formulation that we derive from it in Corollary 5 can also be derived from [6]. Namely, our Integer Programming formulation yields a face of the polytope $Q_{\mathcal{A}}$ of all triangulations as considered by [6]. That is, we get our inequality system of Corollary 5 from $Q_{\mathcal{A}}$ by adding the constraints $x_{\sigma}=0$ for all cones $\sigma \notin \mathcal{C}$ to the description of $Q_{\mathcal{A}}$ given in [6] that uses the "chamber equation." With this, Theorem 4 is a combination of results from Section 3 of [6].

Theorem 4 (Characterization of PARTITIONS). Given a cone $C \subseteq \mathbb{R}^{n}$, a system of subcones $\mathcal{C}=\left\{C^{1}, \ldots, C^{r}\right\}$, the set $\mathcal{F}=\left\{F^{1}, \ldots, F^{s}\right\}$ of their interior facets, and a generic point $g_{0}$, the following statements are equivalent:

1. $\mathcal{C}$ defines a PARTITION of the cone $C$.

2. $g_{0}$ is contained in exactly one of the subcones $C^{i}$, and whenever $F^{j} \in \mathcal{F}$ is a facet of $C^{k} \in \mathcal{C}$ (which is interior for $C$ ), there is a unique second subcone $C^{l} \in \mathcal{C}$ with $C^{k} \cap C^{l}=F^{j}$ such that $F^{j}$ is a facet of $C^{l}$.

3. $g_{0}$ is contained in exactly one of the subcones $C^{i}$, and all interior facets $F^{j}$ are the facets of an even number of $C^{j}$ 's, with "the same number of subcones $C^{j}$ on both sides." 
Proof. $\quad(1) \Rightarrow(2)$ and $(2) \Rightarrow(3)$ are clear.

$(3) \Rightarrow(1)$ The set $\bigcup_{i=1}^{r} \partial C^{i}$ is closed and $(n-1)$-dimensional, thus the set $G$ of all generic points is open and dense in $C$. By construction all the points in any connected component of $G$ are contained in the same number of subcones $C^{i}$.

Claim 1. Every generic point $g \in G$ is contained in exactly one subcone $C^{i}$.

In any small neighborhood $U_{\varepsilon}(g)$ that is contained in $G$ we find a generic point $g^{\prime} \in U_{\varepsilon}(g)$ such that the interval

$$
\left[g_{0}, g^{\prime}\right]:=\left\{x \in C: x=\lambda g_{0}+(1-\lambda) g^{\prime} ; 0 \leq \lambda \leq 1\right\}
$$

is in general position, that is, such that it contains only a finite number of nongeneric points, where it crosses facets of subcones (that is, it does not intersect any faces of subcones of dimension $d<n-1$ ). The nongeneric points of this interval define a segmentation of $\left[g_{0}, g^{\prime}\right]$ into finitely many subintervals and we see by moving from one interval to the next that the interior of each such subinterval is contained in exactly one subcone $C^{i}$. In particular, the point $g^{\prime}$ is contained in exactly one subcone, and thus the same is true for the point $g$, since $g^{\prime}$ and $g$ lie in the same connected component of $G$.

(Figure 1 illustrates the situation, by representing an affine section of a three-dimensional cone. Only the triangles that meet the line $g^{\prime} g_{0}$ are drawn. They meet face-to-face.)

Since $G$ is dense in $C$, Claim 1 also implies that the set $\mathcal{C}$ is a cover of $C$. Now we will show that this cover also is a partition, that is, the intersection of any two subcones is a face of both. For this we note that by Claim 1 the intersection of any two subcones is of dimension at most $n-1$ : otherwise the intersection would contain generic points that are covered at least twice.

Claim 2. Let $C^{i}$ be one of the subcones, let $x$ be a point in the boundary of $C^{i}$, and let $F \subseteq C^{i}$ be the smallest face of $C^{i}$ that contains $x$, such that $x$ is in the relative interior

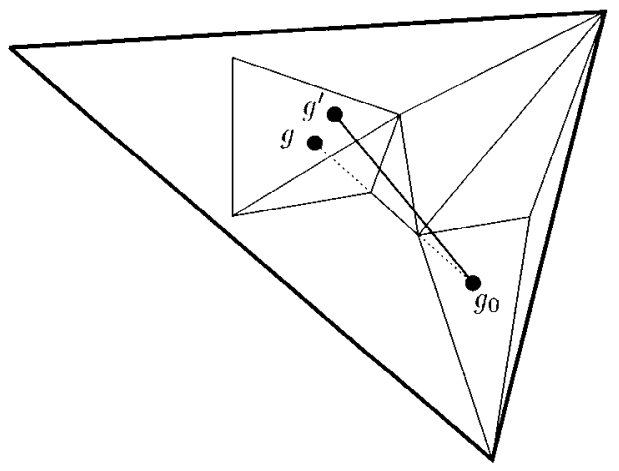

Fig. 1 
of $F$. Then every small enough convex open neighborhood $U=U_{\varepsilon}(x)$ has the property that $U \cap C$ is covered by the subcones $C^{k}$ that have $F$ as a face.

The proof of this claim is analogous to the argument for Claim 1, with the additional observation that for $\varepsilon$ small enough all the faces of $C^{i}$ that meet $U$ must have $F$ as a face, and the same is true for the chain of subcones $C^{j}$ that arises in the argument of Claim 1.

To complete the argument, assume that there are two cones $C^{i}$ and $C^{k}$ such that the intersection $S:=C^{i} \cap C^{k}$ is not a face of $C^{i}$. There is a smallest face $F$ of $C^{i}$ that contains $S$, given as the intersection of all faces of $C^{i}$ that contain $S$. In particular, $S$ contains some relative interior point of $F$, which we take as the point $x$. Now the subcones that contain $x$ and have $F$ as a face cover a small neighborhood of $x$. Thus, since $C^{k}$ contains $x$, it either has $F$ as a face, or it has a full-dimensional intersection with a subcone that has this property. In either case we get a contradiction.

For more details see [7]. Using an integer programming algorithm, this characterization allows one to test the existence of HILBERT PARTITIONS, and to find one if they exist.

Corollary 5 (IP Formulation). Let $C \subseteq \mathbb{R}^{n}$ be a cone as before, let $\mathcal{H}=\left\{h^{1}, \ldots, h^{k}\right\}$ be the minimal Hilbert basis of $C$, let $\mathcal{U}=\left\{C^{1}, \ldots, C^{r}\right\}$ be the set of all unimodular subcones spanned by elements of the Hilbert basis, let $\mathcal{F}=\left\{F^{1}, \ldots, F^{s}\right\}$ be the set of their interior facets, and let $g_{0} \in C$ be a generic point. Then the HILBERT PARTITIONS of $C$ are in bijection with the feasible solution of the integer program

$$
\begin{array}{rlrl}
\min \sum_{j=1}^{r} x_{i}: \sum_{j=1}^{r} \tilde{M}_{0 j} x_{j} & =1, & \\
\sum_{j=1}^{r} \tilde{M}_{i j} x_{j} & =0 & & \text { for } 1 \leq i \leq s, \\
x_{j} & \in\{0,1\} & \text { for } 1 \leq j \leq r,
\end{array}
$$

via

$$
x \quad \longleftrightarrow \mathcal{C}^{x}:=\left\{C^{i} \in \mathcal{U}: x_{i}=1\right\}
$$

For this the matrix $\tilde{M} \in \mathbb{Z}^{(1+s) \times r}$ is given by

$$
\begin{aligned}
& \tilde{M}_{0 j}:= \begin{cases}1, & \text { if } g_{0} \in C^{j}, \\
0, & \text { otherwise, }\end{cases} \\
& \widetilde{M}_{i j}:= \begin{cases}\operatorname{det}\left(h^{i_{t}}, h^{i_{1}}, \ldots, \widehat{h^{i_{t}}}, \ldots, h^{i_{n}}\right), & \text { if } F^{i} \text { is a facet of } C^{j}, \\
0, & \text { otherwise, }\end{cases}
\end{aligned}
$$

for $1 \leq i \leq$ s and $1 \leq j \leq r$, where the subcone $C^{j}$ is given by $C^{j}=\operatorname{cone}\left\{h^{i_{1}}, \ldots, h^{i_{n}}\right\} \in$ $\mathcal{C}$, and $F^{i}$ is one of its facets, represented as $F^{i}=\operatorname{cone}\left\{h^{i_{1}}, \ldots, \widehat{h}^{i_{t}}, \ldots, h^{i_{n}}\right\} \in \mathcal{F}$, with $1 \leq t \leq n$. (Since all subcones are unimodular, the nonzero entries in the matrix $M$ are +1 or -1 , depending on the position of the subcones in relation to the inner facets.) 
Proof. The first equation guarantees that the generic point $g_{0}$ is in exactly one subcone. For every interior facet the system has a corresponding equation, which models the requirement that each inner facet must be in an even number of subcones, with "the same number on both sides." Thus by (1) $\Leftrightarrow$ (3) of Theorem 4 the sets $\mathcal{C}^{x} \subseteq \mathcal{U}$ given by the characteristic vectors $x$ of solutions are the HILBERT PARTITIONS. The objective function selects a HILBERT PARTITION with the minimal number of unimodular subcones.

Theorem 6 (Characterization of BINARY COVERS). Given a cone $C \subseteq \mathbb{R}^{n}$, a system of subcones $\mathcal{C}$, the set $\mathcal{F}$ of interior facets and a generic point $g_{0}$ as above, the following statements are equivalent:

1. The set $\mathcal{C}$ is a BINARY COVER of the cone $C$.

2. $g_{0}$ is contained in an odd number of subcones $C^{i}$, and all inner facets $F^{j}$ are the facets of an even number of subcones $C^{j}$.

Proof. (1) $\Rightarrow(2)$ This is the definition of a binary cover.

$(2) \Rightarrow(1)$ The proof is the same as for Claim 1 in Theorem 4 above: we obtain that if $g_{0}$ is covered an odd number of times, then every generic point $g \in G$ is contained in an odd number of subcones $C^{i}$.

Corollary 7 (GF(2) Formulation). Let $C \subseteq \mathbb{R}^{n}$ be a cone as above, let $\mathcal{U}=$ $\left\{C^{1}, \ldots, C^{r}\right\}$ be the set of all unimodular subcones spanned by elements of the Hilbert basis, let $\mathcal{F}=\left\{F^{1}, \ldots, F^{s}\right\}$ be the set of all interior facets, and let $g_{0} \in C$ be a generic point.

Then the BINARY HILBERT COVERS of $C$ are in bijection with the feasible solutions $x \in(G F(2))^{r}$ of the following linear system of equations over $\mathrm{GF}(2)$ :

$$
\begin{aligned}
\sum_{j=1}^{r} M_{0 j} x_{j} & =1, & & \\
\sum_{j=1}^{r} M_{i j} x_{j} & =0 & & \text { for } 1 \leq i \leq s, \\
x_{j} & \in \mathrm{GF}(2) & & \text { for } \quad 1 \leq j \leq r,
\end{aligned}
$$

via

$$
x \longleftrightarrow \mathcal{C}^{x}:=\left\{C^{i} \in \mathcal{U}: x_{i}=1\right\} .
$$

Here the matrix $M \in(G F(2))^{(1+s) \times r}$ is given by

$$
\begin{aligned}
M_{0 j} & := \begin{cases}1, & \text { if } g_{0} \in C^{j}, \\
0, & \text { otherwise, }\end{cases} \\
M_{i j}: & = \begin{cases}1, & \text { if } F^{i} \text { is a facet of } C^{j}, \\
0, & \text { otherwise, }\end{cases}
\end{aligned}
$$

for $1 \leq i \leq s$ and $1 \leq j \leq r$.

Proof. By definition of the matrix, the first equation models that the generic point $g_{0}$ lies in an odd number of subcones of a binary cover, and the other equations represent 
that every interior facet lies in an even number of subcones. By Theorem 6 the sets $\mathcal{C}^{x} \subseteq \mathcal{U}$ derived from the solutions $x$ of this system define the BINARY HILBERT COVERS.

\section{Examples}

In the following examples, " $C:=$ cone $M$ " for a matrix $M \in \mathbb{Z}^{m \times n}$ denotes the (rational, polyhedral, pointed) cone spanned by the rows of the matrix $M$. In particular, in our first examples we have $m=n$, and thus $C$ is a simplicial cone whose determinant is given by the determinant of $M$. To use even more of a shorthand, we use

$$
C\left[a_{1}, a_{2}, \ldots, a_{n}\right]:=\text { cone }\left(\begin{array}{ccccc}
1 & 0 & \cdots & 0 & 0 \\
0 & 1 & \cdots & 0 & 0 \\
\vdots & \vdots & \ddots & \vdots & \vdots \\
0 & 0 & \cdots & 1 & 0 \\
a_{1} & a_{2} & \cdots & a_{n-1} & a_{n}
\end{array}\right)
$$

for the cone spanned by the first $n-1$ unit vectors together with $a=\left(a_{1}, \ldots, a_{n}\right) \in \mathbb{Z}^{n}$. For $a_{n}>0$ this is a simplicial cone of determinant $a_{n}$. The condition that $\operatorname{gcd}\left(a_{i}, a_{n}\right)=1$ for $1 \leq i<n$ is equivalent to the fact that all the facets of $C\left[a_{1}, \ldots, a_{n}\right]$ are unimodular.

In the following examples, we have tested the existence of HILBERT PARTITIONS via Corollary 5, using $\mathrm{CPLEX}^{1}$ to solve the integer programs that arise. The existence of BINARY HILBERT COVERS was tested via Corollary 7, using our own implementation of a Gauss algorithm to solve the linear systems of equations over GF(2) that arise from it.

The saga of counterexamples in this field starts with $C[1,4,3,7]$, the example of Bouvier and Gonzalez-Sprinberg [2], [3] from 1992, of a four-dimensional cone that does not have a HILBERT PARTITION. A slight simplification yields the following.

Example 8 (No Hilbert Partition). The four-dimensional cone $C[1,2,3,5]$ does not have a Hilbert PARTITION, but it does have a BINARY HilbERT COVER.

However, Lagarias and Ziegler [8] now have infinite classes of cones that have BINARY HILBERT COVERS but no HILBERT PARTITION. Thus here we only present an especially nice example found in our search: a four-dimensional cone whose facets are unimodular.

Definition 9 (Hyperplane Property; Parallelotope Cones). An $n$-dimensional cone $C \subseteq \mathbb{R}^{n}$ has the hyperplane property if its Hilbert basis $\mathcal{H}(C)$ is contained in a hyperplane $H(C)$. Neccessarily this hyperplane must be spanned by the integer generators of the cone. For cones of the form $C=C\left[a^{1}, \ldots, a^{n}\right]$ this hyperplane is given by

$$
H(C)=\left\{x \in \mathbb{R}^{n}: x_{1}+\cdots+x_{n-1}+\frac{1-\left(a_{1}+\cdots+a_{n-1}\right)}{a_{n}} x_{n}=1\right\} .
$$

\footnotetext{
${ }^{1}$ CPLEX Linear Optimizer 4.0.8 with with Mixed Integer \& Barrier Solvers; (c)CPLEX Optimization, Inc., 1989-1995
} 
The hyperplane property is of algebraic interest, since it corresponds to the fact that the corresponding monoid ring is normal. (See, e.g., [4].)

A simplicial cone $C=\operatorname{cone}\left\{b^{1}, \ldots, b^{n}\right\} \subseteq \mathbb{R}^{n}$ is a parallelotope cone if all the nonzero integer points in the half-open parallelogram spanned by the generating vectors

$$
\operatorname{par}(C):=\left\{\lambda_{1} b^{1}+\cdots+\lambda_{n} b^{n} \in \mathbb{R}^{n}: 0 \leq \lambda_{i}<1 \text { for } i=1, \ldots, n\right\}
$$

belong to the Hilbert basis, that is, if we have $|\mathcal{H}|=\operatorname{det}(C)+n-1$. (The terminology is from [9].)

We have searched for cones of the form $C\left[a_{1}, a_{2}, a_{3}, a_{4}\right]$ with the conditions that $0<a_{1}<a_{2}<a_{3}<a_{4}-1<100, \operatorname{gcd}\left(a_{1}, a_{2}, a_{3}, a_{4}\right)=1$, and with the hyperplane property: We found 10 such cones without a HILBERT PARTITION. All of them have a BINARY HILBERT COVER. Only one of them has unimodular facets:

Example 10 (No Hilbert Partition, Hyperplane Property, Unimodular Facets). The four-dimensional simplicial cone $C[14,31,34,39]$ has a BINARY HILBERT COVER, but no Hilbert PARTITION. (Its Hilbert basis has $|\mathcal{H}|=12$ elements; it spans $r=161$ unimodular subcones.) It has the hyperplane property. All its facets are unimodular.

Our extensive search in dimension 4 did not produce any examples of simplicial cones without a BINARY HILBERT COVER. Thus we also investigated this problem in dimension 5 with cones of the form $C\left[a_{1}, a_{2}, a_{3}, a_{4}, a_{5}\right]$, where $a \in \mathbb{Z}^{5}, 0<a_{1}<a_{2}<$ $a_{3}<a_{4}<a_{5}-1$, and $\operatorname{gcd}\left(a_{1}, a_{2}, a_{3}, a_{4}, a_{5}\right)=1$. From our analysis of all such cones with determinant at most $20\left(a_{5} \leq 20\right)$ we found 10 without a BINARY HILBERT COVER. Up to unimodular equivalence these cones give two distinct examples.

Example 11 (No BINARY HILBERT COVER). The five-dimensional simplicial cones

$$
C_{1}:=C[2,3,5,13,19] \quad \text { and } \quad C_{2}:=C[2,5,11,13,19]
$$

have no BINARY HiLberT COVER. Both of them have determinant 19, and both have a Hilbert basis of cardinality 23, so they are parallelotope cones. All their facets are unimodular. $C_{1}$ has $r=7059$ unimodular subcones, $C_{2}$ has $r=7106$ of them.

Currently, we do not know whether these cones do satisfy any of the weaker properties-but computational tests with millions of random points suggest that they do have a HILBERT COVER, and thus in particular that they satisfy the INTEGER CARATHÉODORY PROPERTY.

Ohsugi and Hibi [10], [11] produced two nonsimplicial cones of dimensions 10 and 20 , for which they could show that they are normal (which implies the hyperplane property), and that they have no REGULAR HILBERT PARTITION.

Example 12 (A Hilbert Partition, but no Regular Hilbert Partition). The 10dimensional nonsimplicial cone shown in Fig. 2 has a HILBERT PARTITION, but no REgular Hilbert PARTITION. This cone is generated from a graph on 10 vertices by associating with the edge $\{i, j\}$ the generator $h=e_{i}+e_{j}+e_{10}$ if $10 \notin\{i, j\}$, and 


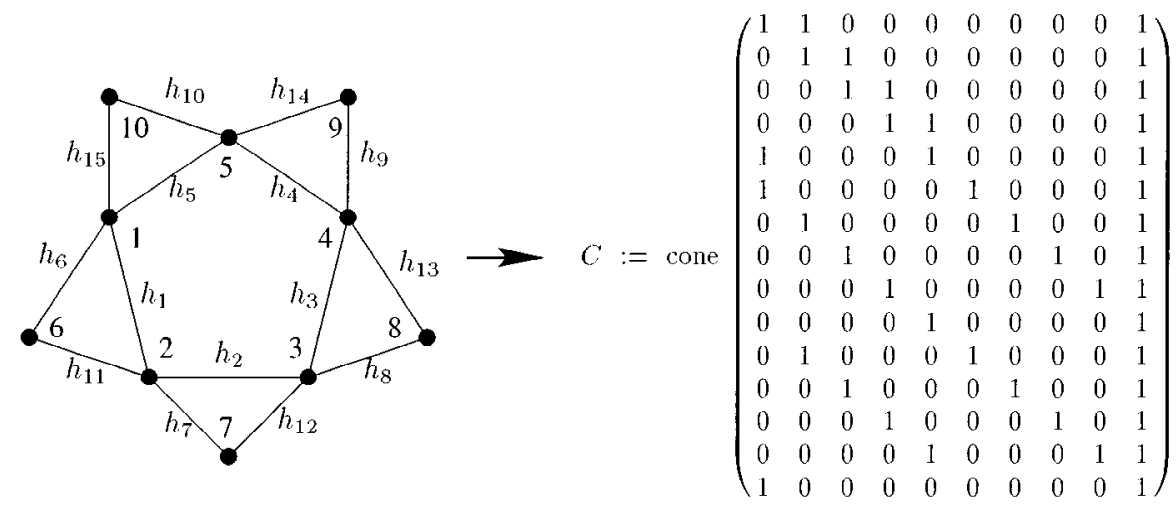

Fig. 2

$h=e_{i}+e_{j}$ otherwise. This cone has the property that the 15 generators (i.e., the rows $h_{1}, \ldots, h_{15}$ of the matrix) form the complete Hilbert basis. In particular, the cone has the hyperplane property, with $H(C)=\left\{x \in \mathbb{R}^{10}: x_{10}=1\right\}$ ). Ohsugi and Hibi [10] have shown that there is no regular unimodular triangulation for this cone. (Their Gröbner basis proof can be translated into a simple geometric argument.)

The HILBERT PARTITION found by CPLEX for this example provides a triangulation containing 56 unimodular subcones (out of $r=1202$ ), and thus the polytope $C \cap H$ has (normalized) volume 56. This coincides with the normalized volume computed by Ohsugi and Hibi from the Gröbner basis of the associated binomial ideal.

A detailed analysis of Ohsugi and Hibi's example yields more remarkable properties. In particular, De Loera confirmed the existence of a HILBERT PARTITION for this example using his program PUNTOS [5], and he has analyzed the complete set of triangulations without new vertices of the polytope $C \cap H$ :

- The triangulation into 56 top-dimensional simplices is unique (up to symmetry); it is nonregular, but it can be connected to a regular tringulation by one single "flip."

- Not only the maximal number of top-dimensional simplices in a triangulation without new vertices (56) cannot be achieved by a regular triangulation; also the triangulation with the minimal number of simplices (46) is unique, but not regular: all regular triangulations have at least 48 simplices.

Subsequently, Ohsugi and Hibi [11] found another example of the same type, a 20dimensional 0/1-cone with 24 generators, for which they found that it does not have a Regular Hilbert Partition. It is derived in the same way as their first example, from the graph shown in Fig. 3. Again, using Corollary 5 we determined that the cone does have a HILBERT PARTITION: the example turns out to have $r=4617$ unimodular cones spanned by the Hilbert basis, 307 of which appear in the solution. (CPLEX had no difficulty with the integer programming problem. The example is too large for PUNTOS.) 


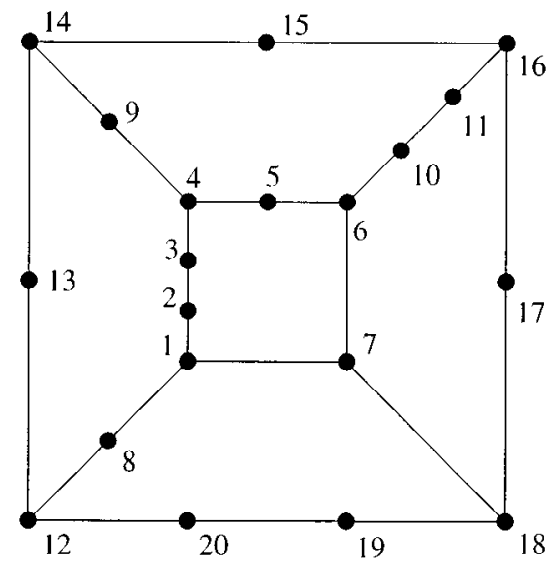

Fig. 3

\section{Acknowledgments}

Thanks to Jesus De Loera, Jörg Rambau, Hidefumi Ohsugi, and Takayuki Hibi for useful conversations related to Example 12. Thanks to Michael Joswig for the HILBERT COVER tests on Example 11.

\section{References}

1. St. Aguzzoli and D. Mundici: An algorithmic desingularization of 3-dimensional toric varieties, Tôhoku Math. J. 46 (1994), 557-572.

2. C. Bouvier and G. Gonzalez-Sprinberg: $G$-desingularisations de varietes toriques, C. R. Acad. Sci. Paris Ser. I 315 (1992), 817-820.

3. C. Bouvier and G. Gonzalez-Sprinberg: Système générateur minimal, diviseurs essentiels et $G$ désingularisations de variétés toriques, Tôhoku Math. J. 47 (1995), 125-149.

4. W. Bruns, J. Gubeladse and N. Viêt Trung: Normal polytopes, triangulations, and Koszul algebras, J. Reine Angew. Math. 485 (1997), 123-160.

5. J. De Loera: Triangulations of polytopes and computational algebra, Ph.D. thesis, Cornell University, 1995. PUNTOS - computation of triangulations of polytopes: available via anonymous ftp at ftp: //geom. umn. edu directory priv/deloera.

6. J. A. De Loera, S. Hoşten, F. Santos and B. Sturmfels: The polytope of all triangulations of a point configuration, Doc. Math. (J. DMV) 1 (1996), 103-119.

7. R. T. Firla: Algorithms for the Hilbert partition and Hilbert cover problems, Diplomarbeit, TU Berlin, 1997.

8. J. Lagarias and G. M. Ziegler: Unimodular triangulations, in preparation.

9. J. Liu: Hilbert bases with the Carathéodory property, Ph.D. Thesis, Cornell, 1991, 79 pages; Preprint 91-3, Computational Optimization Project, Cornell University, 1991.

10. H. Ohsugi and T. Hibi: A normal $(0,1)$-polytope none of whose regular triangulations is unimodular, Preprint, 1997; Discrete Comput. Geom., this issue, pp. 201-204.

11. H. Ohsugi and T. Hibi: Personal communication, June 1997.

12. A. Schrijver: Theory of Linear and Integer Programming, Wiley-Interscience, Chichester, 1986.

13. A. Sebő: Hilbert bases, Carathéodory's theorem and combinatorial optimization, in: Integer Programming and Combinatorial Optimization (R. Kannan and W. Pulleyblank, eds.), Mathematical Programming Society, University of Waterloo Press, Waterloo, 1990, pp. 431-456. 
14. B. Sturmfels: Gröbner Bases and Convex Polytopes, A.M.S. University Lecture Series, Vol. 8, American Mathematical Society, Providence, RI, 1995.

15. G. M. Ziegler: Lectures on Polytopes, Graduate Texts in Mathematics, Vol. 152, Springer-Verlag, New York, 1995; revised printing 1998.

Received September 5, 1997, and in revised form October 10, 1997.

Note added in proof (June 1998). Very recently, Winfried Bruns and Joseph Gubeladze have announced a counterexample to the HILBERT COVER conjecture in dimension $d=6$ : the Hilbert basis has size 12 and satisfies the hyperplane property. The details will be reported in

W. Bruns and J. Gubeladze: Normality and covering properties for affine semigroups, Preprint, 1998. 